\title{
Metal-Linked Dimerization in the Iron-Dependent Regulator from Mycobacterium tuberculosis ${ }^{\dagger}$
}

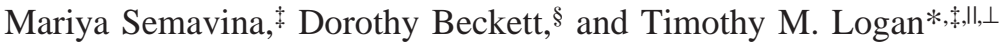 \\ Graduate Program in Molecular Biophysics, Kasha Laboratory, Florida State University, Tallahassee, Florida 32306, \\ Department of Chemistry and Biochemistry and Center for Biological Structure and Organization, University of Maryland, \\ College Park, Maryland 20742, Department of Chemistry and Biochemistry, Florida State University, Tallahassee, Florida \\ 32306, and National High Magnetic Field Laboratory, 1800 E Paul Dirac Drive, Tallahassee, Florida 32310
}

Received April 24, 2006; Revised Manuscript Received July 20, 2006

\begin{abstract}
The iron-dependent regulator (IdeR) is a 230-amino acid transcriptional repressor that regulates iron homeostasis, oxidative stress response and virulence in Mycobacterium tuberculosis. The natural ligand for IdeR is $\mathrm{Fe}(\mathrm{II})$, but $\mathrm{Ni}(\mathrm{II}), \mathrm{Co}(\mathrm{II}), \mathrm{Cd}(\mathrm{II}), \mathrm{Mn}$ (II), and $\mathrm{Zn}$ (II) also bind to and activate the protein in vitro. Protein activation by metal is a complex process involving metal-induced folding of the $\mathrm{N}$-terminal domain, changes in the interaction between the $N$ - and $C$-terminal domains, and the formation of homodimers. Here, we investigate the energetics of dimerization and metal binding in IdeR. The dimerization energetics were determined as a function of metal binding using equilibrium analytical ultracentrifugation. The equilibrium dimer dissociation constant of apo-IdeR was $4.0 \mu \mathrm{M}$ at $20^{\circ} \mathrm{C}$. The dissociation constant decreased to $0.5 \mu \mathrm{M}$ in the presence of one equivalent of $\mathrm{Ni}(\mathrm{II}) \mathrm{Cl}_{2}$ and decreased further $\left(K_{\mathrm{d}} \ll 50 \mathrm{nM}\right)$ in the presence of excess Ni(II). IdeR contains two tryptophan residues. The addition of $\mathrm{Ni}$ (II) induced changes in fluorescence intensity and emission maximum of the tryptophan residues that strongly depended on protein concentration. At low IdeR concentration, fluorescence was enhanced at low metal-to-protein ratios but was quenched at high metal-to-protein ratios. At high IdeR concentration, metal binding resulted only in fluorescence quenching. The fluorescence enhancement at low protein concentrations was buffer-dependent and required the presence of both tryptophans. Metal binding affinity was measured quantitatively using equilibrium dialysis. The results showed strongly positive cooperative binding of three equivalents of metal per monomer with an average apparent dissociation constant of 2.2 $\pm 0.3 \mu \mathrm{M}$ and a Hill coefficient of 2. Metal binding was not cooperative in an IdeR variant that showed reduced affinity for dimer formation. The results of this study establish the positive cooperative nature of metal binding by IdeR and suggest that dimerization is a major contributor to cooperative binding. The strong coupling between metal binding and dimerization places specific constraints on the activation mechanism.
\end{abstract}

Iron is an essential element for most living organisms, including bacteria, where it plays a critical role in metabolism, proliferation, and infection. Ide ${ }^{1}$ facilitates the adaptation of Mycobacteria to iron limitation encountered by the pathogen in the host cell. IdeR is a dual functional regulator

$\dagger$ This work was supported by grants from the National Institutes of Health (Grant R01 AI021628), the Florida State University Research Foundation (to T.M.L.), and by National Institutes of Health Shared Instrumentation Program (Grant S10-RR15899 to D.B.).

* Corresponding author. Tel: 850-644-8979. Fax: 850-644-7244. E-mail: logan@sb.fsu.edu.

$\doteqdot$ Graduate Program in Molecular Biophysics, Florida State University.

University of Maryland.

"Department of Chemistry and Biochemistry, Florida State University.

${ }^{\perp}$ National High Magnetic Field Laboratory.

${ }^{1}$ Abbreviations: IdeR, iron-dependent regulator from Mycobacterium tuberculosis; DtxR, diphtheria toxin repressor from Corynebacterium diphtheriae; HEPES, 4-(2-hydroxyethyl)piperazine-1-ethanesulfonic acid; Tris, tris(hydroxymethyl)aminomethane; gshIdeR, IdeR containing $\mathrm{N}$-terminal Gly-Ser-His tripeptide; SH3, protein domain having structural homology to the third domain of src; DTT, dithiothreitol; EDTA, ethylenediaminetetraacetic acid; IPTG, isopropyl $\beta$-D-thiogalactopyranoside; DLS, dynamic light scattering; terpy, $2,2^{\prime}, 2^{\prime \prime}$-terpyridine. that acts under iron-rich conditions as a repressor of siderophore biosynthesis genes ( $m b t A, m b t B, m b t I)$ and as an activator of iron storage $(b f r A, b f r B)(1)$ and oxidative stress response $(k a t G, \operatorname{sod} A)(2,3)$ genes. In addition, IdeR controls genes encoding proteins involved in general metabolism and virulence determinants (4). The diversity of cellular processes controlled by IdeR requires the finely tuned sensing of available iron levels, and IdeR appears to be the central element in this complex network. Null mutations in the ideR gene are lethal (5), further highlighting its importance in M. tuberculosis.

Initial studies of IdeR and its closest homologue, the diphtheria toxin repressor (DtxR) from Corynebacterium diphtheriae, indicated two metal binding sites. However, a recent high-resolution structure of IdeR $(6,7)$ and metal binding studies of DtxR (8) are consistent with more than two equivalents of metal bound per monomer. One metal binding site is formed entirely by the residues from the $N$-terminal domain (M10, C102, E105, and H106) (Figure 1). Mutating any one of these residues to alanine abrogates repressor activity in DtxR (9); this binding site is referred to as the 


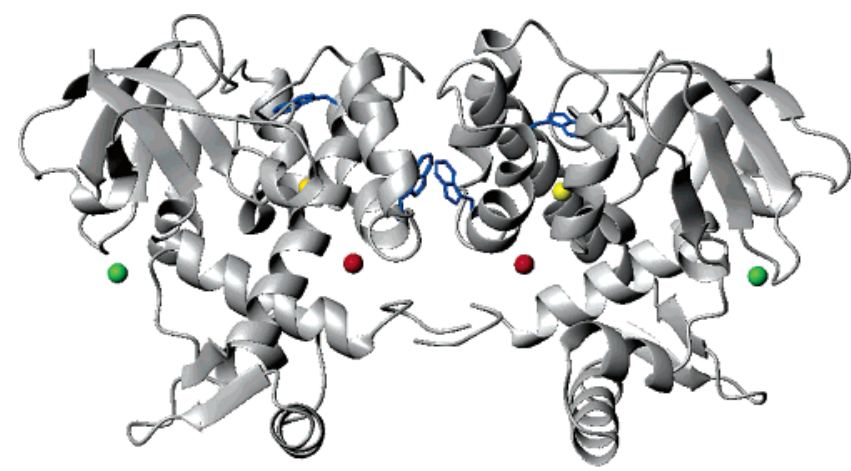

FIGURE 1: Crystal structure of the holo-IdeR dimer. Metal ions bound to site 1 (yellow), site 2 (red) and site 3 (green) are shown. Tryptophan residues are shown in blue. The Figure was generated from the 1U8R pdb file (6) using Molmol (48).

primary metal binding site. A second metal binding site is formed by residues from the $\mathrm{N}$-terminal domain and by two residues from the $C$-terminal SH3-like domain (H79, E83, H98, E172, and Q175). Mutating any one of these residues in DtxR reduces but does not abolish repressor activity (9); consequently, this site is referred to as the ancillary site. The primary and ancillary metal binding sites are separated by $\sim 9 \AA$ and interact via a series of hydrogen bonds and salt bridges in the metal bound form (10), which suggests that a functional cooperativity may exist between these two sites. The recently identified third metal binding site in IdeR is located on the surface of the SH3-like $C$-terminal domain, coordinated by $\mathrm{H} 219, \mathrm{H} 223$, and solvent water (6); the biological significance of this site has not yet been established. In DtxR, residue $\mathrm{H} 219$ participates in the interaction between the $C$-terminal domain and DNA-binding domain by making contact with E21 (11); however, in IdeR structures, these two residues are over $9 \AA$ apart $(6,7)$.

IdeR binds the inverted repeat operator sequence on duplex DNA as a fully metalated dimer of dimers $(6,7)$. In the absence of metal, IdeR, like DtxR (12), exists as a partially folded, weakly dimeric species. The proposed model of protein activation suggests that metal binding induces folding of the $N$-terminal domain and enhances dimer formation. Previous studies of DtxR have confirmed metal-induced dimerization (13). Here, we characterize the molecular processes leading to activation of DNA binding in IdeR by directly measuring the energetics of dimerization and metal binding. The linkage between dimerization and metal binding was investigated by sedimentation equilibrium experiments. The dimerization affinity increases upon binding the first equivalent of metal. Binding additional metal equivalents has a stronger affect on the dimerization. Equilibrium dialysis experiments reveal cooperative binding of two equivalents of $\mathrm{Ni}$ (II) per monomer with a possible third equivalent of bound metal. Cooperative metal binding is strongly dependent on dimer formation, as metal binding in a construct of IdeR that has a reduced ability to dimerize was not cooperative. The metal binding and dimerization studies are consistent with a multistep model of activation of apo-IdeR by transition metal ions.

\section{MATERIALS AND METHODS}

Construction of Expression Vectors. The IdeR(wt) construct in the pTrc vector was a gift from Dr. J. R. Murphy
(Boston University Medical School, Boston, MA). The cDNA for the IdeR insert was subcloned into the pET11b or $\mathrm{pET} 15 \mathrm{~b}$ vector (Novagen, Madison, WI) by digesting the plasmid at unique Bam HI and HindIII restriction sites and ligating it into an identically digested empty vector.

IdeR tryptophan mutants W94F and W104F were generated using a QuickChange mutagenesis kit (Stratagene, La Jolla, CA) and the following primers: W94F forward, 5'CGGGTTGCCGTTCGAAGAAGTTCACGCCGAGGC; W94F reverse, 5'-GCCTCGGCGTGAACTTCTTCGAACGGCAACCCG; W104F forward, 5'-CGCCGAGGCATGCCGGTTCGAGCACGTGATGAGCG; and W104F reverse, 5'-CGCTCATCACGTGCTCGAACCGGCATGCCTCGGCG.

Protein Purification and Demetalation. IdeR, IdeR(W94F), and IdeR(W104F) were expressed in $E$. coli BL21/DE3 terrific broth media using an overnight auto-induction protocol (Novagen, Madison, WI). Cells were harvested by centrifugation, and the pellets were resuspended in $50 \mathrm{mM}$ Tris and $40 \mathrm{mM} \mathrm{NaCl}$ at $\mathrm{pH} 8.5$ (buffer A), lysed using a microfluidizer (Microfluidics Corporation, Newton, MA) according to the manufacturer's instructions, and clarified by centrifugation. The clarified cell lysate was loaded on a $\mathrm{Ni}^{2+}$-NTA affinity column (HisTrap) preequilibrated with buffer A, and the protein was eluted using a linear gradient of buffer B (50 mM Tris, $40 \mathrm{mM} \mathrm{NaCl}, 100 \mathrm{mM}$ imidazole at $\mathrm{pH}$ 8.5). Fractions containing IdeR were pooled, concentrated, and chromatographed over a Q-sepharose anion-exchange column (all chromatography columns were from Amersham Biosciences, Piscataway, NJ). IdeR eluted between 0.25 and 0.30 $\mathrm{M} \mathrm{NaCl}$. IdeR-containing fractions were concentrated and exchanged into $10 \mathrm{mM}$ HEPES, $2 \mathrm{M} \mathrm{NaCl}$, and $3 \mathrm{M}$ Urea ( $\mathrm{pH}$ 5.5) and chromatographed on a Phenyl-sepharose hydrophobic interaction column using a linear gradient from 2 to $0 \mathrm{M} \mathrm{NaCl}$. IdeR eluted in two fractions, at 0 and $2 \mathrm{M} \mathrm{NaCl}$. Both fractions were pooled and concentrated, and EDTA was added at $20 \mathrm{mM}$ final concentration. Protein was refolded and demetalated by exhaustive dialysis against $10 \mathrm{mM}$ HEPES at pH 6.8 in the presence of CHELEX 100. Protein concentration was measured spectrophotometrically in the presence of $6 \mathrm{M}$ urea at $280 \mathrm{~nm}$ (14) using a calculated extinction coefficient of $14000 \mathrm{~cm}^{-1} \mathrm{M}^{-1}$ (www.expasy.org) for wild-type IdeR and $8250 \mathrm{~cm}^{-1} \mathrm{M}^{-1}$ for IdeR(W94F) and IdeR(W104F). All subsequent steps were performed in acidwashed glassware using CHELEX-treated buffers. The purification scheme resulted in IdeR samples that exhibited minimal batch-to-batch variation in various biophysical probes including fluorescence and UV-vis spectra, DNA binding, and ITC profiles.

IdeR with a removable $N$-terminal hexahistidine affinity tag was expressed from $E$. coli transformed with the pET15bIdeR plasmid. Cells were harvested by centrifugation and lysed as above. The debris was pelleted and the supernatant applied on a $\mathrm{Ni}^{2+}$-NTA affinity column preequilibrated with $50 \mathrm{mM}$ sodium phosphate containing $50 \mathrm{mM}$ imidazole and $0.5 \mathrm{M} \mathrm{NaCl}$ (binding buffer). The protein was eluted using $50 \mathrm{mM}$ sodium phosphate containing $0.5 \mathrm{M}$ imidazole and $0.5 \mathrm{M} \mathrm{NaCl}$ (elute buffer). Fractions containing IdeR were pooled and subjected to thrombin cleavage $(0.1 \mathrm{U}$ per $1 \mathrm{mg}$ of protein) for $16 \mathrm{~h}$ at $25^{\circ} \mathrm{C}$. The extent of cleavage was confirmed by SDS-PAGE. The cleaved protein was then unfolded in $6 \mathrm{M}$ urea and $20 \mathrm{mM}$ EDTA, and refolded in 
metal-free $10 \mathrm{mM}$ HEPES at $\mathrm{pH}$ 6.8. IdeR prepared in this manner contained a Gly-Ser-His tripeptide at the $N$-terminus, and is referred to here as gshIdeR.

Activation for Metal Binding. IdeR contains a single Cys residue that slowly forms intermonomer disulfide bonds that inactivate the protein. Prior to metal binding experiments, the disulfide was reduced by treatment with $5 \mathrm{mM}$ dithiothreitol for $60 \mathrm{~min}$ at $37^{\circ} \mathrm{C}$. DTT was removed by passing the sample through a HiTrap desalting column at $1 \mathrm{~mL} / \mathrm{min}$ and exchanging the buffer to metal-free $10 \mathrm{mM}$ HEPES at $\mathrm{pH}$ 6.8. Free cysteine content in the reduced IdeR was quantified using Ellman's reagent (15) prior to metal binding experiments. In the experiments reported here, the number of free cysteines per IdeR monomer averaged $0.98 \pm 0.06$ (expected number is 1), with no change in this ratio following the metal binding experiments. IdeR samples with ratios $<0.95$ were not used for metal binding or dimerization experiments. IdeR samples reduced under these conditions were capable of DNA binding (as evidenced by the gel mobility shift assays) for up to $72 \mathrm{~h}$ (longest time tested). The glassware used for all procedures after reduction and desalting were soaked in 17\% nitric acid overnight and rinsed rigorously with Nanopure water.

Fluorescence Spectroscopy. Tryptophan fluorescence was monitored from 290 to $450 \mathrm{~nm}$, with excitation at $280 \mathrm{~nm}$ at room temperature $(12,16,17)$. Apo-protein was titrated with $1-2 \mu \mathrm{L}$ aliquots from 1 or $10 \mathrm{mM} \mathrm{Ni(II)Cl} l_{2}$ solutions. Fluorescence scans were taken $5 \mathrm{~min}$ after each addition of metal. Fluorescence quenching was analyzed by integrating the area under each curve and plotting fractional changes in areas versus the metal/protein ratio. Each experiment was performed as an average of at least three independent experiments.

Dynamic Light Scattering. The apparent hydrodynamic radius of IdeR and gshIdeR were measured using a DynaPro instrument (Protein Solutions, Bucks, England) at $20{ }^{\circ} \mathrm{C}$. Protein solutions $(1-2 \mathrm{mg} / \mathrm{mL})$ were prepared in metal-free $10 \mathrm{mM}$ HEPES and $100 \mathrm{mM} \mathrm{NaCl}$ at $\mathrm{pH} 6.8$ with (holosamples) or without (apo-samples) the addition of 10-fold excess of $\mathrm{NiCl}_{2}$. Samples were passed through a $0.2 \mu \mathrm{m}$ filter and centrifuged for $15 \mathrm{~min}$ at $15000 \mathrm{rpm}$ to eliminate dust particles and large aggregates prior to DLS experiments. The molecular masses were estimated from the measured radii by applying a power law relation for globular proteins (MW $=1.68 R_{\mathrm{H}}{ }^{2.34}$, where the hydrated radius, $R_{\mathrm{H}}$, is given in $\mathrm{nm}$ ). The populations of different species were calculated from the amplitudes of the respective components of the correlation functions as an average of 20 measurements (10 acquisitions each), and the errors were calculated as a standard deviation from the mean value.

Equilibrium Dialysis. Equilibrium dialysis experiments were performed at $25^{\circ} \mathrm{C}$ using $1 \mathrm{~mL}$, two-compartment equilibrium dialysis cells (BEL-ART Scienceware, Pequannock, NJ) with 3500 Da MWCO membrane and airtight seals. The membranes were soaked overnight in a CHELEX-treated buffer prior to each experiment. In a typical dialysis experiment, $800 \mu \mathrm{L}$ of a reduced, demetalated protein solution at $30 \mu \mathrm{M}$ was placed on one side of the dialysis cell while the same volume of $\mathrm{Ni}(\mathrm{II}) \mathrm{Cl}_{2}$ in the same buffer was placed on the other side. Equilibrium was reached within 16-18 h. The concentration of free $\mathrm{Ni}(\mathrm{II})$ in each cell was determined using $2,2^{\prime}, 2^{\prime \prime}$-terpyridine (terpy) (18), which exhibits char- acteristic absorbance bands at 320 and $334 \mathrm{~nm}$ (19) when bound to $\mathrm{Ni}(\mathrm{II})$. All samples were placed in $5 \mathrm{M}$ urea to ensure the release of all metal from the protein, and terpy was added to a final concentration of $330 \mu \mathrm{M}$. The amount of metal in each sample was calculated on the basis of the area under terpy-Ni(II) absorption bands using a calibration curve generated from $\mathrm{Ni}$ (II) standards (SPEX CertiPrep, Metuchen, NJ). The binding function $(r)$ was calculated as

$$
r=\frac{N i_{\mathrm{t}}^{2+}-N i_{\mathrm{f}}^{2+}}{P_{\mathrm{t}}}
$$

where $\mathrm{Ni}_{\mathrm{t}}{ }^{2+}$ and $\mathrm{Nif}_{\mathrm{f}}{ }^{2+}$ are $\mathrm{Ni}^{2+}$ concentrations on the protein and protein-free side, respectively, and $P_{\mathrm{t}}$ is the total protein concentration. Data analysis was performed using Origin 6.0 software, and the best fits were obtained to a binding function assuming $n$ sites with infinite cooperativity and apparent equilibrium dissociation constant $K_{\mathrm{D}}$ as follows.

$$
r=\frac{n x^{\mathrm{n}}}{K_{\mathrm{D}}^{\mathrm{n}}+x^{\mathrm{n}}}
$$

The binding isotherms obtained from equilibrium dialysis experiments with gshIdeR were fitted to a noncooperative ligand binding model described by the following equation:

$$
r=\frac{n x}{K_{\mathrm{D}}+x}
$$

where $n$ is the number of identical independent sites, and $K_{\mathrm{D}}$ is the apparent equilibrium dissociation constant for these sites.

Sedimentation Equilibrium. Dimerization energetics of apo, partially metal bound, and fully metal bound IdeR were measured by analytical ultracentrifugation using an Optima XL-I analytical ultracentrifuge (Beckman Coulter, Inc., Fullerton, CA) equipped with a four-hole An60 Ti rotor. Double sector cells with charcoal-filled Epon centerpieces with an optical path length of $12 \mathrm{~mm}$ and quartz windows were used. The sample cell contained $140 \mu \mathrm{L}$ of protein with or without metal, whereas the reference cell contained 155 $\mu \mathrm{L}$ of dialysis buffer. Protein concentration distributions generated during centrifugation were determined from absorption at $280 \mathrm{~nm}$. Absorbance scans were taken after $18 \mathrm{~h}$ at each speed and represent the average of three measurements at each radial position with a spacing of $0.001 \mathrm{~cm}$. For partially saturated IdeR, an aliquot of $\mathrm{NiCl}_{2}$ was added to make the final ratio of metal to protein $0.8: 1$; holo-IdeR was prepared with 10 -fold molar excess metal over protein. All three sets of samples were prepared at 12, 24, and 40 $\mu \mathrm{M}$ protein concentration, and centrifugation was performed at 22000,26000 , and $30000 \mathrm{rpm}$ and additionally at 18000 rpm for holo-IdeR.

Data were analyzed using the NONLIN program (20) adapted for the analysis of sedimentation equilibrium data (MacNONLIN). Initially, sedimentation equilibrium data were analyzed using a model for a single homogeneous species described by the following equation:

$$
c_{\mathrm{r}}=\delta c+c_{0} \exp \left\{\frac{M(1-\bar{v} \rho) \omega^{2}\left(r^{2}-r_{0}{ }^{2}\right)}{2 R T}\right\}
$$


A
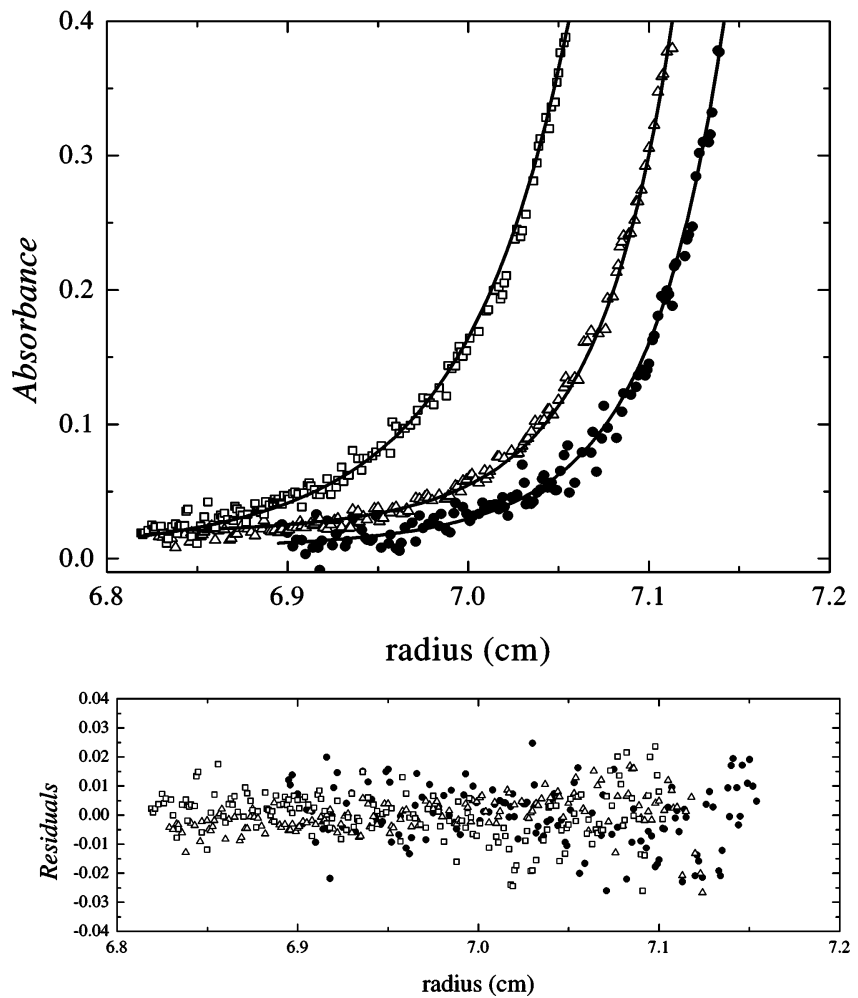

B
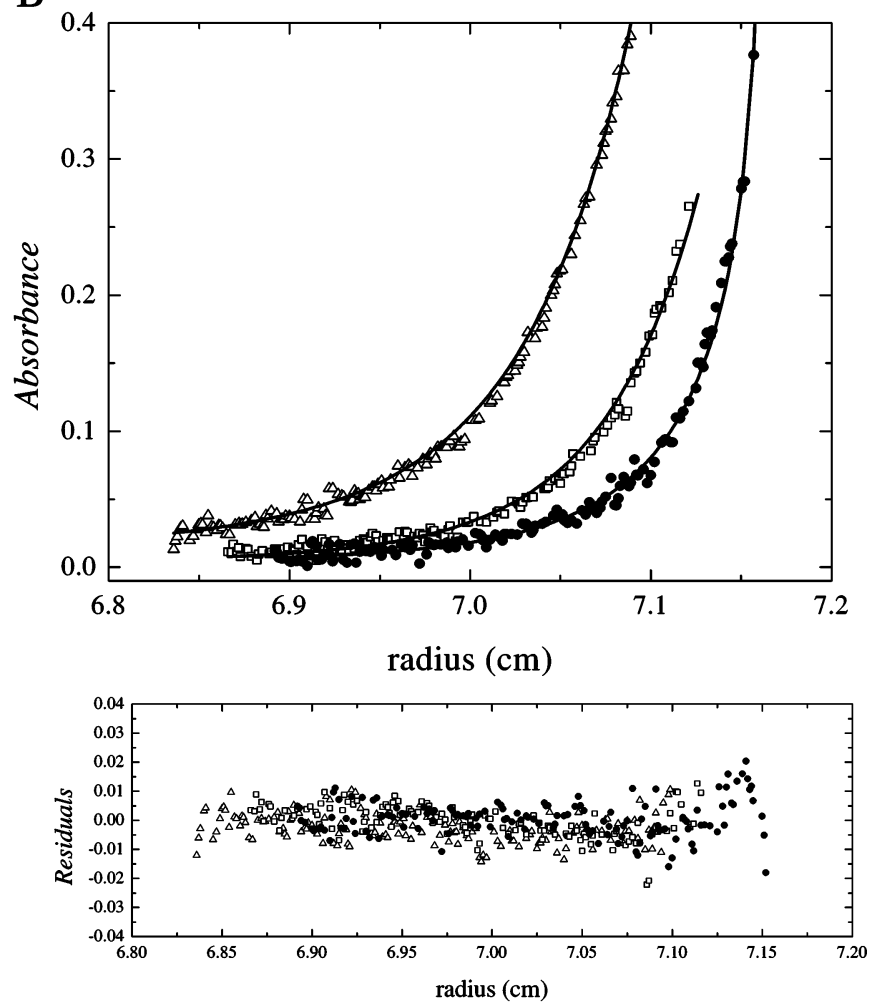

FIgURE 2: Analytical ultracentrifugation of IdeR. (A) apo-IdeR centrifuged at $12 \mu \mathrm{M}$ and $26000 \mathrm{rpm}(\triangle), 24 \mu \mathrm{M}$ and $26000 \mathrm{rpm}(\triangle)$, $40 \mu \mathrm{M}$ and $22000 \mathrm{rpm}(\mathbf{O})$. (B) IdeR at a loading concentration of $24 \mu \mathrm{M}$ centrifuged at $22000 \mathrm{rpm}$ in the presence of $0(\triangle), 20 \mu \mathrm{M}(\square)$, and $200 \mu \mathrm{M}\left(\mathbf{O} \mathrm{NiCl}_{2}\right.$. The solid lines in $\mathrm{A}$ and $\mathrm{B}$ represent the best fit obtained from a global analysis of nine datasets to a monomerdimer model (eq 6).

where $c_{\mathrm{r}}$ is the total protein concentration at a given radial position, $c_{0}$ is the protein concentration at a reference position (first point in the dataset), $M$ is the molecular mass of the protein, $\bar{v}$ is the partial specific volume of the protein (determined to be 0.742 for the apo-IdeR monomer using the SEDNTERP program (http://www.rasmb.bbri.org)), $\rho=$ $1.002 \mathrm{~g} / \mathrm{mL}$ is the solvent density, $\omega$ is the angular velocity of the rotor, $r$ is the radial position in centimeters from the center of rotation, $r_{0}$ is the radial distance of the reference position, $R$ is the gas constant, $T$ is the absolute temperature, and $\delta c$ is a correction term for baseline offset. Here, the data were fit to the reduced buoyant molecular mass as defined by the following equation:

$$
\sigma=\frac{M(1-\bar{v} \rho) \omega^{2}}{R T}
$$

where the symbols are the same as those indicated above. Dimerization equilibrium constants were determined from a global analysis of datasets collected at multiple loading concentrations and multiple rotor speeds based on the following model:

$$
\begin{aligned}
& c_{\mathrm{r}}=c_{\mathrm{m}}\left(r_{0}\right) \exp \left\{\frac{\sigma\left(r^{2}-r_{0}{ }^{2}\right)}{2}\right\}+ \\
& K_{\mathrm{a}} c_{\mathrm{m}}{ }^{2} \exp \left\{\frac{\sigma\left(r^{2}-r_{0}{ }^{2}\right)}{2}\right\}
\end{aligned}
$$

where $c_{\mathrm{m}}$ is the monomer concentration and $K_{\mathrm{a}}$ is the monomer-dimer equilibrium association constant. The
Table 1: Assembly Properties of IdeR Protein at Different Ligation States as Measured by Sedimentation Equilibrium

\begin{tabular}{lcc}
\hline \multicolumn{1}{c}{ sample } & $\begin{array}{c}\text { apparent }^{a} \\
\text { MW }(\mathrm{Da})\end{array}$ & $\begin{array}{c}\text { monomer- } \operatorname{dimer}^{b} K_{\mathrm{d}} \\
(\mu \mathrm{M})\end{array}$ \\
\hline apo IdeR & 42187 & $4 \pm 2$ \\
IdeR + 1 eq. metal & 47382 & $0.5 \pm 0.3$ \\
holo IdeR & 58579 & beyond detection limit \\
\hline
\end{tabular}

${ }^{a}$ Molecular masses were calculated from the fit of nine datasets to an ideal single species model. The calculated molecular weight of the monomer is $25234 \mathrm{Da}^{b}$ Equilibrium dimerization constants were obtained from a global fit of nine datasets to a monomer-dimer association model.

criteria for the quality of fit were the distribution of the residuals and the magnitude of the square root of the variance of the fits.

\section{RESULTS}

Dimerization Energetics in IdeR. The association energetics of IdeR and its dependence on metal binding were determined by a series of sedimentation equilibrium experiments performed at multiple rotor speeds and a range of loading concentrations. We first determined the dimerization constant for apoIdeR by comparing the sedimentation results obtained at different protein concentrations and rotor speeds (Figure 2A). Global analysis of the resulting distributions to eq 6 yielded an equilibrium dissociation constant for dimer formation in the low micromolar range (Table 1), consistent with a previous article on DtxR (13). In a second set of experiments, we investigated the linkage between metal 

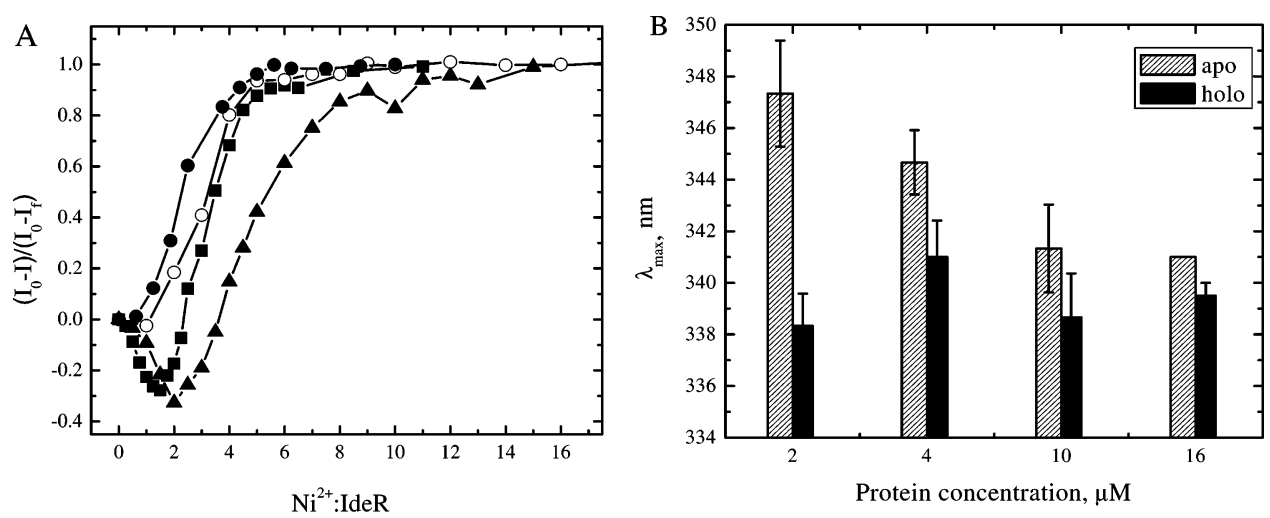

FIGURE 3: Fluorescence quenching by $\mathrm{Ni}(\mathrm{II}) \mathrm{Cl}_{2}$ in IdeR at different concentrations. (A) Normalized fluorescence as a function of the

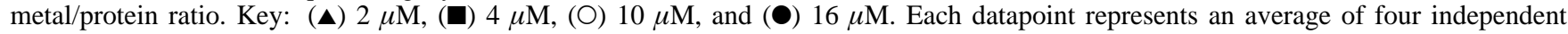
experiments. The error bars are not shown for clarity but were never higher than $10 \%$ of the mean. The solid line is drawn to guide the eye and does not represent a fit to a binding model. (B) Changes in $\lambda_{\max }$ upon metal binding.

binding and dimer formation. As seen in Figure 2B, the addition of one equivalent of $\mathrm{Ni}(\mathrm{II}) \mathrm{Cl}_{2}$ increased the amount of dimer present, whereas saturation with metal resulted in further enhancement. Quantitative analysis of the resulting curves using eq 6 showed that dimer affinity increases by nearly 1 order of magnitude in the presence of one metal equivalent. In the presence of an excess of metal, the dimer affinity is increased beyond the limits of detection of the instrument (Table 1). Indeed, this curve was modeled adequately by assuming a single species corresponding to dimeric IdeR, for example, there was no detectable monomeric species present. The absorption optics employed for the protein detection limited measurements at lower protein concentrations.

Metal Binding in IdeR Depends on Protein Concentration. IdeR contains two tryptophans per monomer. Trp 104 is located at the dimer interface and becomes shielded from the solvent in the dimer, whereas Trp 94 is located toward the top of the $N$-terminal domain and is solvent exposed in the holorepressor (Figure 1). Both tryptophans are close to the primary and ancillary metal binding sites and are expected to be quenched by transition metals upon coordination (16, 21). Thus, we expected that fluorescence quenching would provide a sensitive and quantitative measure of metal binding.

Fluorescence quenching experiments were performed using $\mathrm{Ni}(\mathrm{II}) \mathrm{Cl}_{2}$ at protein concentrations of $2,4,10$, and $16 \mu \mathrm{M}$. A complete titration was performed at each protein concentration, revealing changes in total fluorescence intensity and emission $\lambda_{\max }$. The observed intensity changes are plotted as the normalized change in fluorescence versus the molar ratio of metal to protein in Figure 3A. At low protein concentrations, for example, $2 \mu \mathrm{M}$, metal binding by IdeR resulted in an initial increase in fluorescence (represented by negative quenching values) that was followed by saturable quenching at higher metal-to-protein ratios. As the protein concentration increased, the magnitude of the metal-induced fluorescence enhancement was reduced until at and above $10 \mu \mathrm{M}$ IdeR there was no fluorescence enhancement, and metal binding resulted only in fluorescence quenching (Figure 3A). The biphasic fluorescence change upon metal binding precluded a quantitative analysis of the binding isotherms over an extended range of protein concentration. Nevertheless, the data in Figure 3A indicate a progressive increase in the apparent metal binding affinity as the protein concentration is increased: saturation of the $2 \mu \mathrm{M}$ sample occurs when approximately 7 equivalents of metal are added, whereas the $16 \mu \mathrm{M}$ sample is saturated after 4 equivalents.

During metal induced activation, IdeR undergoes conformational ordering and dimerization $(12,16)$; these processes can affect tryptophan fluorescence intensity and the emission maximum. We, therefore, expected to see metal-associated changes of fluorescence intensity and $\lambda_{\max }$ of the emission. The emission $\lambda_{\max }$ of apo and metal saturated IdeR at four different protein concentrations is shown in Figure 3B. The $\lambda_{\max }$ for holoIdeR was essentially independent of protein concentration, whereas $\lambda_{\max }$ for apoIdeR was blue shifted upon increasing protein concentration and by metal binding. The blue shift indicates a transition to a less polar tryptophan environment, consistent with the burial of tryptophans associated with dimer formation in the aporepressor. To test this hypothesis, we plotted the $\lambda_{\max }$ for apoIdeR over an extended concentration range $(2-80 \mu \mathrm{M})$ and superimposed on it the fraction dimer calculated from the apo-IdeR dimer dissociation constant in Table 1 (Figure 4A), yielding an excellent correlation.

Because the W94 side chain is solvent exposed in the dimeric metal bound repressor, we assumed that the blue shift represents changes in the W104 environment, which is located at the dimer interface (Figure 1). We made mutants in which either tryptophan residue was mutated to phenylalanine and measured the $\lambda_{\max }$ as a function of protein concentration (Figure 4B). Emission from W94 (as measured in the W104F mutant) showed minimal change in $\lambda_{\max }$ up to $80 \mu \mathrm{M}$ apoIdeR; in contrast, tryptophan emission from W104 (as measured in the W94F mutant) showed a protein concentration dependence that mimicked the wild-type protein (Figure 4B), supporting our hypothesis. It should be noted that fluorescence intensity in the apo form of both tryptophan mutants was approximately one-half of that of the wild-type protein, suggesting that it is tryptophan fluorescence that dominates the emission in IdeR, with little, if any, contribution from other potential fluorophores.

To establish the relationship between dimer formation and changes in fluorescence intensity, the results were extended by analyzing the fraction dimer found at specific $\mathrm{Ni}$ (II)/IdeR ratios for each protein concentration. Using the dimer dissociation constant values for apo and partially saturated proteins, we calculated that IdeR contains $34 \%$ dimer at $2 \mu \mathrm{M}$; the fraction of dimer increases to $77 \%$ upon binding 

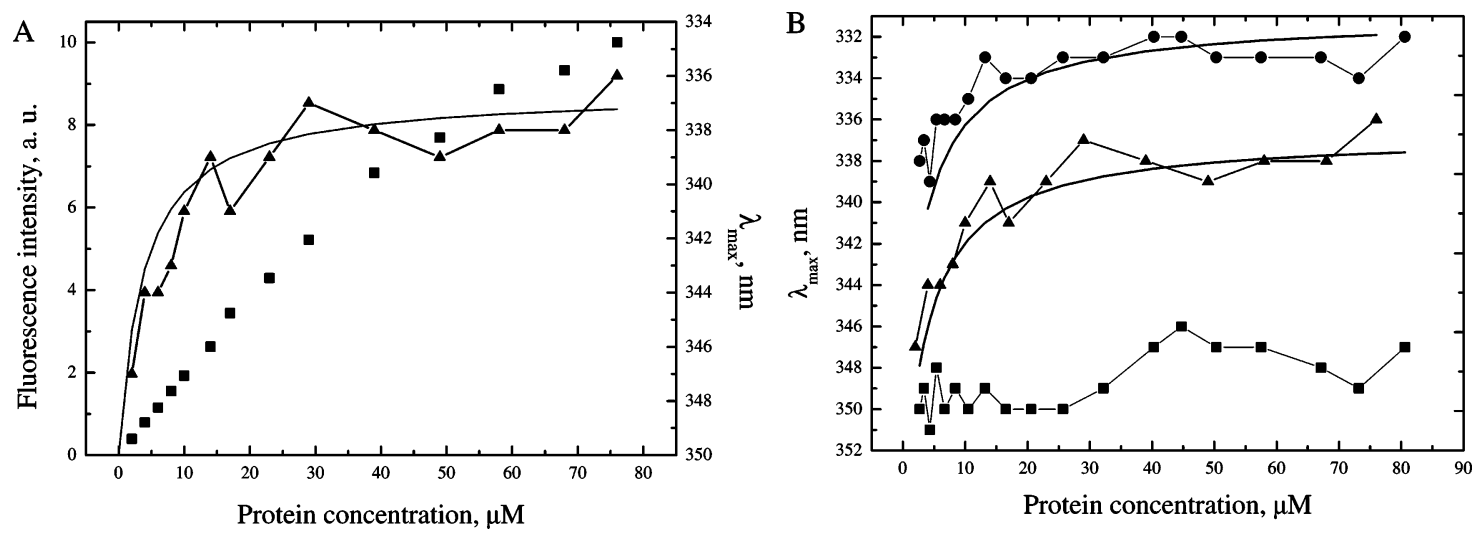

FIGURE 4: Dependence of fluorescence $\lambda_{\max }$ and intensity on the dimer fraction. (A) Wild-type apoIdeR $\lambda_{\max }(\boldsymbol{\Delta})$ and fluorescence intensity

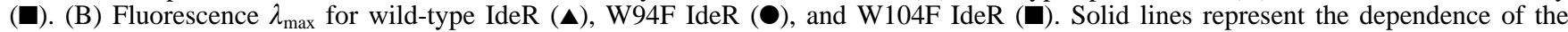
dimer fraction upon protein concentration calculated using the dimerization data in Table 1 .
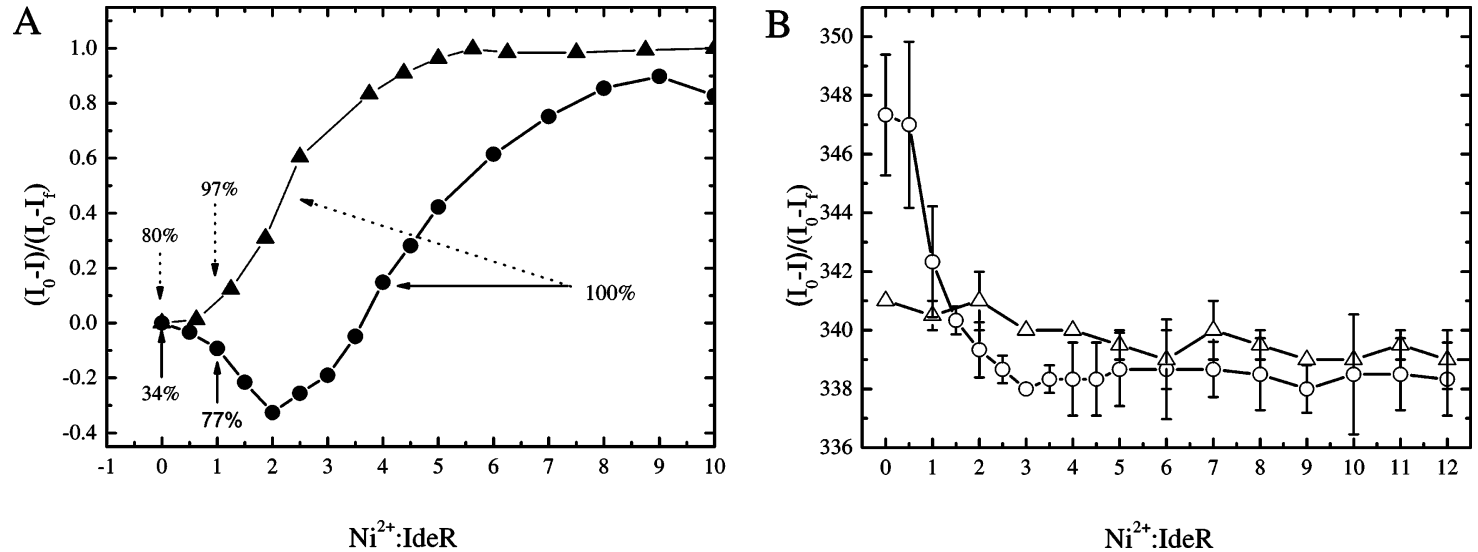

FIGURE 5: Fluorescence parameters as a function of metal concentration for different IdeR concentrations. (A) Metal induced fluorescence intensity changes for $2 \mu \mathrm{M}(\boldsymbol{\bullet}$, solid line arrows) and $16 \mu \mathrm{M}(\boldsymbol{\Delta}$, dotted line arrows) IdeR. The amount of dimer was calculated using equilibrium sedimentation fit results from Table 1. (B) Metal induced changes in $\lambda_{\max }$ for $2 \mu \mathrm{M}(\mathrm{O})$ and $16 \mu \mathrm{M}(\Delta)$ IdeR.

the first metal, and reaches $100 \%$ at saturation (Figure 5A). The apo-state of the $16 \mu \mathrm{M}$ IdeR sample is composed of $80 \%$ dimer; this number approaches $100 \%$ as the first metal is bound. Combining this with the information presented in Figure 5, we estimate that fluorescence quenching occurs when at least $75 \%$ of the protein is dimeric and that the fluorescence enhancement is associated with the monomeric form. In support of this, we see a definite correlation between the blue shifting of the emission $\lambda_{\max }$ and increase in fluorescence intensity upon metal binding. As demonstrated in Figure 5, both the $\lambda_{\max }$ blue shift and fluorescence enhancement for the $2 \mu \mathrm{M}$ sample exhibit greatest change when the first two equivalents of metal are added. At $16 \mu \mathrm{M}$, however, the change in $\lambda_{\max }$ is minimal, and no fluorescence enhancement is observed.

To address the metal induced changes in the fluorescence intensity of the individual tryptophans, $\mathrm{Ni}$ (II) titrations were performed at different protein concentrations. We anticipated that W104F IdeR would not show fluorescence enhancement at low protein concentration, whereas W94F would. However, both mutants exhibited similar levels of fluorescence enhancement at low protein concentrations that was lower than that in the wild-type IdeR (not shown). The reduced enhancement seen in the two single-tryptophan mutants could reflect a mutation induced change in the conformational averaging in apoIdeR such that the fluorescence properties of the remaining tryptophans are altered. Alternatively, the reduced enhancement in the single-tryptophan mutants may suggest the presence of electronic coupling between the two tryptophans. Additional evidence for electronic coupling of the two tryptophans may come from the shifted $\lambda_{\max }$ for W94F (338 nm) and W104F (351 nm) compared to the $\lambda_{\max }$ for the wild type (346 nm).

Gly-Ser-His Extension Decreases Dimer Formation. The apparent linkage between dimer formation and metal binding was investigated using gshIdeR. The three residue $N$-terminal Gly-Ser-His extension (gsh) that remains upon the removal of the hexahistidine affinity tag was previously shown to negatively affect dimerization of DtxR (12), resulting in an inactive repressor even in the metal-bound form (12). Here, we show that the $N$-terminal extension also reduces dimer formation in apo-IdeR. Wild-type apo-IdeR at $70 \mu \mathrm{M}$ exhibited a two-component distribution with Stokes radii of 2.09 and $3.08 \mathrm{~nm}$ in a dynamic light scattering (DLS) experiment. These radii correspond to estimated molecular weights of 19 and $47 \mathrm{kDa}$, respectively. Because the molecular mass of (monomeric) IdeR is $25.2 \mathrm{kDa}$, the DLS data were interpreted as reflecting a monomer-dimer equilibrium in apoIdeR, with populations of $16 \pm 7 \%$ monomer and $79 \pm 7 \%$ dimer calculated from the amplitude of the components of the correlation function. The addition of excess metal to this sample resulted in the formation of a single species with a 

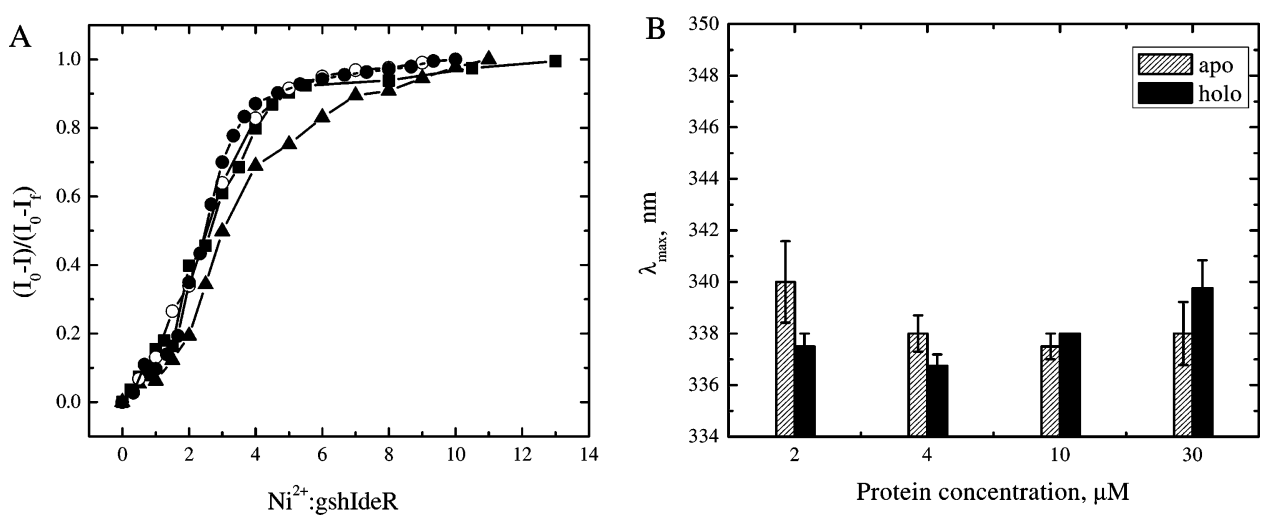

FIGURE 6: Fluorescence quenching by $\mathrm{Ni}(\mathrm{II}) \mathrm{Cl}_{2}$ in gshIdeR at different concentrations. (A) Normalized fluorescence as a function of the metal/protein ratio. Key: (৫) $2 \mu \mathrm{M},(\square) 4 \mu \mathrm{M},(\mathrm{O}) 10 \mu \mathrm{M},(\bullet) 30 \mu \mathrm{M}$. Each datapoint represents an average of four independent experiments. The error bars are not shown for clarity but were never higher than $10 \%$ of the mean. The solid line is drawn to guide the eye and does not represent a fit to a binding model. (B) Changes in $\lambda_{\max }$ upon metal binding.
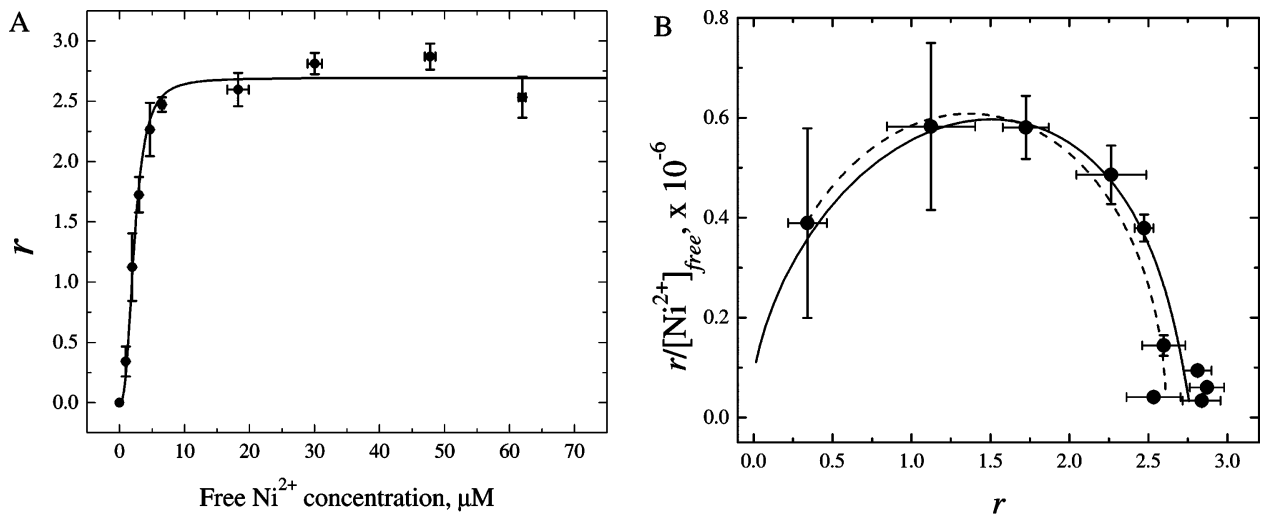

FIGURE 7: Metal binding by IdeR from equilibrium dialysis. (A) Plot of fraction bound as a function of free Ni(II) for IdeR at $30 \mu \mathrm{M}$. Each data point is the average of three independent experiments, and the error bars were calculated as the standard deviation of the mean. The solid line is a fit to eq 2. (B) Scatchard plot of the same ligand binding data. The solid line is a Scatchard transform of eq 2, and the dashed line is the fit to eq 7 .

Stokes radius of $3.08 \mathrm{~nm}(47 \mathrm{kDa})$ indicating the formation of exclusively dimeric IdeR, consistent with a recent article (16).

The gshIdeR mutant also exhibited a species distribution in solution at the same concentration as that of the wildtype IdeR. Two forms with hydrodynamic radii of $2.6 \mathrm{~nm}$ (calculated mass $31 \mathrm{kDa})$ and $3.8 \mathrm{~nm}(76 \mathrm{kDa})$ were observed and were assigned to a monomer and dimer, respectively. Although these values differ from that of the wild type, single site mutations were found to cause similar changes in the hydrodynamic radius of apo-IdeR in an unrelated study (16). In gshIdeR, the $N$-terminal extension may lead to a larger Stokes radius and greater solvent exposed surface area, resulting in a higher apparent molecular weight in the DLS experiments. In contrast to the wild-type IdeR, apo-gshIdeR was predominantly monomeric $(75 \pm 3 \%$ monomer and 24 $\pm 3 \%$ dimer at $70 \mu \mathrm{M}$ gshIdeR). The addition of excess metal increased the fraction of dimer to only $41 \pm 2 \%$, indicating a reduced tendency for metal binding or dimer formation compared to that of the wild-type IdeR.

Metal Binding in gshIdeR Is Independent of Protein Concentration. $\mathrm{Ni}$ (II) titrations resulted in a fluorescence quenching pattern for gshIdeR that differed from that of wild-type IdeR. First, as shown in Figure 6A, metal binding resulted only in fluorescence quenching; fluorescence enhancement was not observed at any protein concentration. Second, the apparent metal binding affinity was independent of protein concentration up to and including $30 \mu \mathrm{M}$. Finally, $\lambda_{\max }$ of apo gshIdeR was blue shifted relative to wild-type apo IdeR at all concentrations and showed smaller blue shifts upon metal binding or increasing protein concentrations (Figure $6 \mathrm{~B})$. Indeed, metal binding may induce a slight red shift at the highest gshIdeR concentrations investigated.

Together, the fluorescence and DLS results show that gshIdeR is capable of binding metal but that metal binding is independent of protein concentration, in contrast to wildtype IdeR. These results suggest that metal binding in gshIdeR does not induce dimer formation to the same extent as that in wild-type IdeR. The mechanism for reduced dimer affinity in gshIdeR or gshDtxR is not clear but probably reflects an increased energetic cost associated with burying the longer $\mathrm{N}$-termini of the two monomers, which are directly apposed in the dimer interface of the holo-repressor (Figure 1).

Quantitative Analysis of Ni(II) Binding in IdeR. Metal binding by IdeR and gshIdeR were quantitatively measured by equilibrium dialysis. This approach allowed us to concentrate on metal binding without the complications of conformational change or dimerization that were encountered in the fluorescence experiments. Total $\mathrm{Ni}$ (II) in each chamber was quantified spectrophotometrically using $2,2^{\prime}, 2^{\prime \prime}$-terpyridine (terpy) $(18,19)$. The fraction of $\mathrm{Ni}(\mathrm{II})$ bound as a function of free $\mathrm{Ni}$ (II) is plotted in Figure $7 \mathrm{~A}$ for IdeR at 30 $\mu \mathrm{M}$. The same data are replotted in the Scatchard plot in Figure 7B. The downward curvature of the Scatchard plot 

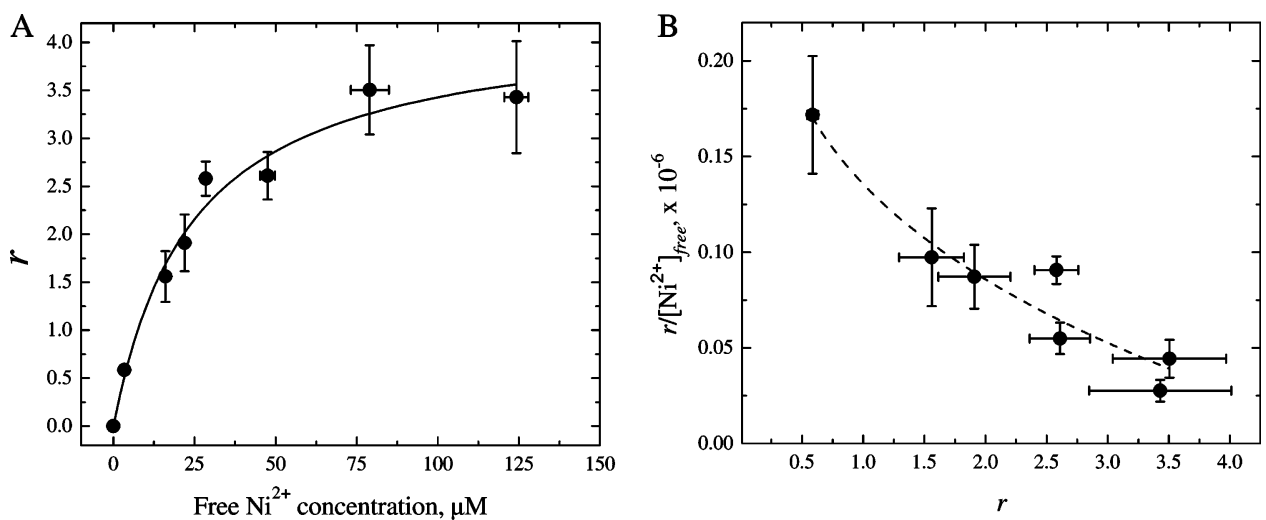

FIGURE 8: Metal binding by gshIdeR from equilibrium dialysis. (A) Plot of fraction bound as a function of free Ni(II) for gshIdeR at $30 \mu \mathrm{M}$. Each data point is the average of four independent experiments, and the error bars were calculated as a standard deviation of the mean. The solid line is a fit to eq 3. (B) Scatchard plot of the same ligand binding data. The dashed line represents the fit to eq 7.

Table 2: Thermodynamic Parameters for Equilibrium Metal Binding to $\operatorname{IdeR}^{a}$

\begin{tabular}{ccc}
\hline protein & $n$ & $k_{\mathrm{D}}, \mu \mathrm{M}$ \\
\hline IdeR & $2.7 \pm 0.2$ & $2.3 \pm 0.3$ \\
gshIdeR & $4.3 \pm 0.3$ & $24.4 \pm 4.9$ \\
\hline${ }^{a}$ For the wild-type IdeR, $n$ is the Hill coefficient. For gshIdeR, $n$ is \\
the number of independently binding sites.
\end{tabular}

is indicative of strong positive cooperativity for ligand binding $(22,23)$. Equilibrium dialysis experiments were performed on gshIdeR, and the results are presented in direct and Scatchard plots in Figure 8. The binding function for gshIdeR differs from that of wild-type IdeR and is consistent with four independent metal binding sites with a single apparent equilibrium dissociation constant that is approximately 1 order of magnitude weaker than that of wild-type IdeR (Table 2). Plotting the data in a Scatchard form yielded a linear plot, indicating noncooperative metal binding.

\section{DISCUSSION}

Metal Binding in IdeR Is Highly Cooperative. The energetics and stoichiometry of metal binding by DtxR and IdeR have been a topic of interest for more than a decade. Initial crystal structures clearly indicated two metal binding sites per monomer in either protein $(7,9,10,24-26)$. The functional significance of these sites for repressor function have been thoroughly investigated in DtxR (9, 27-29). More recently, a third metal binding site located in the SH3 domain was identified in the crystal structure of $\operatorname{IdeR}(6,7)$, although the biological relevance of this site has not been established.

Spiering and colleagues demonstrated that DtxR binds $\mathrm{Ni}(\mathrm{II})$ with a stoichiometry of 2.5 , indicating a possible third binding site (13). Rangachari et al. (30) confirmed that DtxR bound more than 2 equivalents of $\mathrm{Ni}$ (II) per monomer and that the binding appeared to be cooperative. Chou et al. were the first to report positive cooperative metal binding for IdeR (16). In contrast, a recent article (31) argues for noncooperative $\mathrm{Ni}$ (II) binding in DtxR, with high affinity $\left(1.7 \times 10^{-7}\right.$ $\mathrm{M})$ and low affinity $\left(6.4 \times 10^{-4} \mathrm{M}\right)$ binding. Unfortunately, the stoichiometry for these sites was not reported, and therefore, it is difficult to compare their results with the literature or with the data presented here. In this article, we confirm the positive cooperative nature of $\mathrm{Ni}$ (II) binding by IdeR using equilibrium dialysis experiments and provide insights into the molecular origin of the cooperativity.
Plotting the fraction bound as a function of free $\mathrm{Ni}$ (II) for IdeR at $30 \mu \mathrm{M}$ showed that IdeR bound multiple equivalents of $\mathrm{Ni}(\mathrm{II})$. Scatchard transformation clearly indicated the positive cooperative nature of $\mathrm{Ni}$ (II) binding. To further analyze the titrations, we attempted to parametrize the binding by model fitting. We first considered models that describe cooperative binding in terms of a monomer $\leftrightarrow$ dimer equilibrium with either state having unspecified apparent binding affinities and stoichiometries (32). We were able to fit the data with these models, but the confidence intervals for the fitted parameters were large even when the dimer association parameters from Table 1 were used (not shown).

However, simpler models involving simultaneous binding to multiple sites provided improved fits and confidence intervals. As shown in Figure 7A, the equilibrium dialysis data can be fit to a maximally cooperative binding model, for example, one in which partially ligated forms are unpopulated, with a single apparent dissociation constant $K_{\mathrm{D}}$ $=2.3 \mu \mathrm{M}$ and Hill coefficient $=2.7$. The results indicate that IdeR binds roughly 3 equivalents of $\mathrm{Ni}(\mathrm{II})$, which is consistent with the recent high resolution crystal structures $(6,7)$.

The concave downward curvature of the Scatchard plot establishes the positive cooperative nature of $\mathrm{Ni}$ (II) binding by IdeR. Consequently, the data could be modeled adequately by the Scatchard transform of the model with maximal cooperativity (Figure 7B). The Scatchard plot for wild-type IdeR binding data was further analyzed according to eq 7 (33)

$$
\ln \left(\left[L_{\text {free }}\right]\right)=-\frac{1}{\alpha^{\mathrm{H}}} \ln \left[\frac{n}{r}-1\right]+\ln K_{\mathrm{D}}
$$

which describes the behavior of cooperative system in terms of the total number of sites, $n$, the number of interacting sites, $\alpha^{\mathrm{H}}$, and the apparent dissociation constant, $K_{\mathrm{D}}$. This analysis yielded $\alpha^{\mathrm{H}}=2, n=2.6$, and $K_{\mathrm{D}}=2.1 \mu \mathrm{M}$ (Figure $7 \mathrm{~B}$, dashed line), implying that two sites bind in a cooperative fashion, whereas the third site binds independently. A binding model consisting of maximally cooperative sites plus an independent site fits the data equally well; however, the improvement in fit using the more complex model is not statistically significant, given the low number of data points in these experiments (Supporting Information, Figure 1). Nevertheless, the latter model has the conceptual appeal that the cooperative metal binding sites $\left(K_{\mathrm{D}}=2.2 \mu \mathrm{M}\right.$; Hill coefficient $=2$ ) can be assigned to the two sites in the 
$N$-terminal domain, whereas the third, independent site $\left(K_{\mathrm{D}}\right.$ $=4 \mu \mathrm{M}$ ) represents binding by the $\mathrm{SH} 3$ domain. Additional experiments using metal binding site mutants are currently being performed to test this interpretation and will be reported elsewhere. Fitting the Scatchard plot for gshIdeR data to eq 7 (Figure 8B) yielded the number of interacting sites $=0.8$, confirming the lack of cooperativity of metal binding in this construct.

Metal Binding and Dimerization Are Linked in the Activation of IdeR. Metal binding is known to increase the fraction of dimeric protein in DtxR and IdeR, although the detailed mechanism for this remains obscure. We recently demonstrated that DtxR binds metal into the primary metal binding site followed by binding into the ancillary site (30), which led us to propose a mechanism for DtxR activation that rationalizes the biological activity of several metal binding mutants. A key aspect of this mechanism is that dimerization is expected to be enhanced incrementally upon metal binding. This hypothesis was tested here with IdeR by determining the equilibrium constant for dimer dissociation at different $\mathrm{Ni}$ (II)/IdeR ratios. We found that the dimer affinity increased by approximately 1 order of magnitude upon binding the first equivalent of metal, with a larger increase in affinity upon binding the second equivalent. Sedimentation curves collected for apo and 1:1 IdeR/Ni(II) contained both monomeric and dimeric species, whereas the sedimentation profile for IdeR in excess $\mathrm{Ni}$ (II) contained only the dimer. The dissociation data for $\mathrm{Ni}$ (II) saturated IdeR are fit to an equilibrium dissociation constant of $\sim 50 \mathrm{nM}$, but in the absence of a detectable fraction of the monomer, this should be considered an upper limit with the true dimer affinity being higher.

Our results also show that dimerization is involved in the mechanism for cooperative metal binding. First, Ni(II) binding curves for wild-type IdeR shift progressively to higher affinity with increasing protein concentrations. At the lower protein concentration, there is a significant fraction of monomer in the apo form (roughly 66\%) that decreases as the metal binding progresses. In contrast, at the higher protein concentration, corresponding to approximately $80 \%$ dimer in the apo form, metal binding occurs with a significantly higher affinity. Second, although there is strong interaction between the sites in the wild type, all sites bind metal independently in gshIdeR, a predominantly monomeric construct (Table 2). Finally, metal binding in wild-type IdeR is strongly cooperative, whereas metal binding in gshIdeR is not cooperative (Table 2). This provides strong support that dimerization is a major contributor to the strongly positive cooperative metal binding.

The biological role for dimerization as a regulatory mechanism in the activation of IdeR and DtxR is not clear because metal binding is not energetically linked to dimer formation in single-domain members of the DtxR family, such as MntR (34), AntR (34), and MDR1 (35). These latter proteins contain a more extensive dimerization interface and are dimeric even in the apo-state. The ferric uptake regulator, Fur, a functional homologue of DtxR proteins (36), binds DNA as a dimeric protein, but dimerization appears to be linked to $\mathrm{Zn}$ (II) binding rather than to $\mathrm{Fe}(\mathrm{II})(37,38)$, the physiologically relevant metal ion. The dimerization state of other transition and heavy metal binding repressors (MerR, NikR, SmtB/ArsR, and CmtR) also appears to be largely independent of metal binding (39-41), although metal binding induces structural changes that alter DNA binding affinity in these proteins.

Metal Binding and Fluorescence Emission in IdeR. The biphasic nature of the fluorescence emission at low protein concentrations was an unanticipated and striking feature of the fluorescence detected metal binding experiments. Tryptophan fluorescence emission is a sensitive measure of the local environment, which makes it a powerful tool for biophysical studies of proteins. However, this same sensitivity can complicate molecular interpretation of the observed fluorescence changes. In the case of IdeR and other members of the DtxR family, fluorescence emission is quenched in the metal bound form $(16,21)$. This quenching could result from the bound metal, whereas dimer formation $(13,21)$ and stabilization of the $N$-terminal domain (12) may increase or decrease the tryptophan fluorescence emission. The situation in IdeR is further complicated by the presence of two tryptophan residues, W94 and W104, having different solvent exposed surface areas in the metal bound dimer $\left(30\right.$ vs $5 \AA^{2}$, respectively, calculated (42) for pdb file $1 \mathrm{FX} 7$ (7)) and different distances to the metal binding sites of IdeR (Figure 1). Consequently, fluorescence emission in IdeR exhibits a complex pattern that depends on both metal ligation and protein concentration.

One aspect of the fluorescence change is an $\sim 10 \mathrm{~nm}$ blue shift that is induced by metal binding or by increasing protein concentration. The blue shift indicates a change to a less polar environment for one or both tryptophans. We showed that the blue shift is observed when the protein undergoes dimerization, whether due to an increase in protein concentration or due to metal binding. Specifically, W104 showed large dimerization associated changes in $\lambda_{\max }$, whereas W94 was minimally affected. This result agrees strongly with a structure-based prediction for different contributions of the two tryptophans to the fluorescence emission in IdeR: being located on the dimerization surface, W104 is expected to transit to less polar environment upon dimer formation and exhibit blue shifted emission. This result also indicates that the $\lambda_{\max }$ is a sensitive indicator of dimer fraction in apoIdeR. However, we found that the total fluorescence intensity did not correlate with the fraction dimer in apo-IdeR, as suggested previously (16).

We also observed a biphasic change in fluorescence intensity induced by metal binding in wild-type IdeR at low protein concentration. Specifically, metal binding increased total fluorescence at low metal/protein ratios but decreased fluorescence at higher metal/protein ratios. The magnitude of the initial fluorescence enhancement diminished as the protein concentration increased until there was no detectable fluorescence enhancement at $16 \mu \mathrm{M}$ IdeR. The fluorescence enhancement upon metal binding is not observed at any protein concentration in DtxR $(17,21)$, which has a single tryptophan at position W104, nor was it reported in a previous study of IdeR (16).

The primary difference between this work and the previous article (16) is the buffer; here, we used $10 \mathrm{mM}$ HEPES compared with the $10 \mathrm{mM}$ Tris. Ni(II) titrations performed in Tris and HEPES buffer at identical IdeR concentrations ( 2 and $4 \mu \mathrm{M}$ ) and $\mathrm{pH}$ confirmed the absence of fluorescence enhancement in the Tris buffer (not shown). Metal binding by IdeR represents a competition between the protein and 

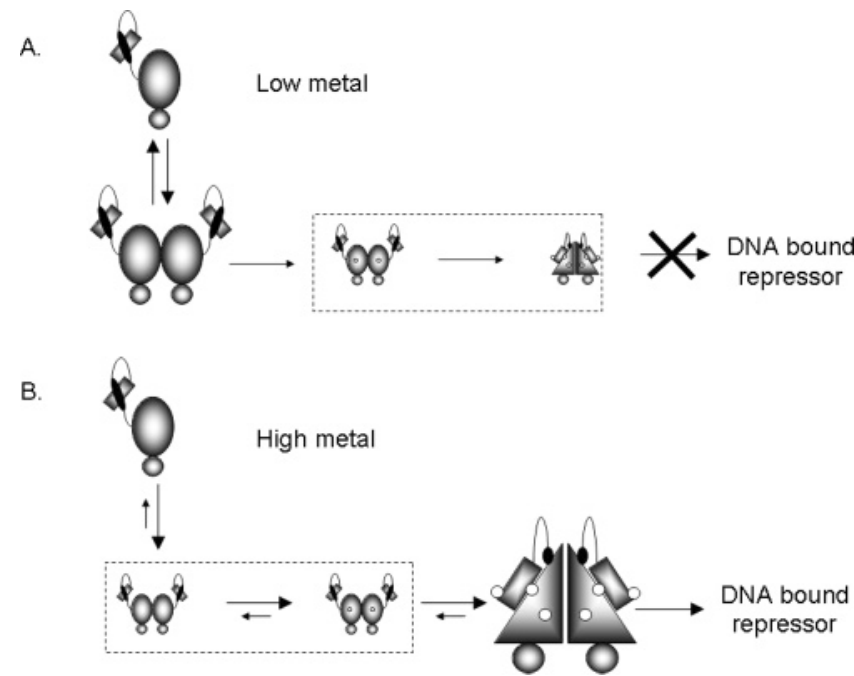

FIGURE 9: Schematic representation of the activation of IdeR. (A) In the apo-form, IdeR exists in monomer $\leftrightarrow$ dimer equilibrium. The SH3-like $C$-terminal domain binds the Pr segment (black rod). Active species of IdeR are not anticipated to be significantly populated and are contained in the box. (B) Upon addition of metal, metal binding (open circles) induces the rearrangement of the $N$-terminal domain (triangle) and shifts the monomer $\leftrightarrow$ dimer equilibrium completely to the dimer form. The Pr segment (black circle) becomes bound to the back of the dimer interface. Strong energetic linkage between metal binding and dimerization results in a very low population of partially ligated monomeric and dimeric species.

small molecule buffer components for the coordination of the solvated metal ions. HEPES was introduced by Good as a nonchelating buffer (43) and is a preferred buffer for $\mathrm{Ni}(\mathrm{II})$-based immobilized metal affinity chromatography because it has a lower binding affinity for $\mathrm{Ni}(\mathrm{II})$ than does Tris, which binds Ni(II) very weakly (44). However, given the low buffer concentration used in this and the previous study (16), buffer coordination would not significantly affect the metal binding equilibrium or alter the sequence of metal binding. Instead, we hypothesize that the ensemble of states forming apo-IdeR is dependent upon buffer conditions such that the fluorescence emission response differs in Tris and HEPES.

Activation of IdeR. The data presented here provide insights into the activation of IdeR, including the dimerization energetics for the apo, holo, and partially metal bound intermediates, establishing the strong positive cooperative nature of metal binding and demonstrating that dimerization is required for cooperative binding. Chou et al. proposed a comprehensive multistep mechanism for the activation of IdeR (16). Briefly, apoIdeR exists as an ensemble of monomers and dimers in equilibrium; the binding of the first metal induces the formation of stable half-holo dimers. Sequential binding of the second metal to each monomer within a dimer results in full activation of the repressor.

The strong coupling between metal binding and dimerization identified in this study constrains the number and nature of possible intermediates in this activation scheme and can be used to simplify the Chou mechanism as shown in Figure 9. In our model, apo-IdeR exists in both monomeric and dimeric forms (Figure 9A). In the effective absence of activating metal, there is no active repressor and sequencespecific DNA binding does not occur. As metal concentration is raised, metal binding can occur from both the monomeric and dimeric forms, leading to an active repressor. Binding the first metal equivalent by monomeric IdeR shifts the equilibrium toward the dimer, where the higher metal binding affinity leads to a metal activated repressor (Figure 9B). The cooperativity between metal binding and dimerization indicates an all-or-nothing metal binding behavior, which implies that IdeR cycles between a fully metalated dimer and demetalated monomers with a partially ligated species essentially unpopulated.

It is important to relate this mechanism for IdeR activation to the protein environment in vivo, given that solution conditions inside live cells differ considerably from the experimental conditions used in this study. The crowded intracellular environment could potentially affect the dimerization by shifting toward the more compact, dimeric state (45). In addition, the in vivo specificity for Fe(II) differs from the observed promiscuity in metal binding in vitro in part through a slightly enhanced affinity for $\mathrm{Fe}(\mathrm{II})$ over Ni(II) $(13,16)$ but also from the relative amounts of free (or available for binding (46)) intracellular $\mathrm{Ni}(\mathrm{II})$ and $\mathrm{Fe}(\mathrm{II})$ (47), directly affecting the on-rate for metal binding. Therefore, the activation mechanism indicated in Figure 9 is expected to be valid in vivo, differing only in the position of the indicated equilibria.

\section{ACKNOWLEDGMENT}

We thank Donald Caspar of Florida State University and Jack Murphy and Robert Harrison of Boston University for stimulating discussions. We thank Ms. Sumiko Takahashi of Florida State University for cloning wild-type IdeR and gshIdeR, and Ms. Saranga Naganathan of the University of Maryland for help in setting up the analytical ultracentrifugation experiments.

\section{SUPPORTING INFORMATION AVAILABLE}

IdeR equilibrium binding data and fits to different models. This material is available free of charge via the Internet at http://pubs.acs.org.

\section{REFERENCES}

1. Gold, B., Rodriguez, G. M., Marras, S. A. E., Pentecost, M., and Smith, I. (2001) The Mycobacterium tuberculosis IdeR is a dual functional regulator that controls transcription of genes involved in iron acquisition, iron storage and survival in macrophages, Mol. Microbiol. 42, 851-865.

2. Dussurget, O., Rodriguez, M., and Smith, I. (1996) An ideR mutant of Mycobacterium smegmatis has derepressed siderophore production and an altered oxidative-stress response, Mol. Microbiol. 22, $535-544$

3. Dussurget, O., and Smith, I. (1998) Interdependence of mycobacterial iron regulation, oxidative-stress response and isoniazid resistance, Trends Microbiol. 6, 354-358.

4. Camacho, C. J., and Thirumalai, D. (1993) Kinetics and thermodynamics of folding in model proteins, Proc. Natl. Acad. Sci. U.S.A. 90, 6369-6372.

5. Rodriguez, G. M., Voskuil, M. I., Gold, B., Schoolnik, G. K., and Smith, I. (2002) ideR, an essential gene in Mycobacterium tuberculosis: Role of IdeR in iron-dependent gene expression, iron metabolism, and oxidative stress response, Infect. Immun. 70, 3371-3381.

6. Wisedchaisri, G., Holmes, R. K., and Hol, W. G. J. (2004) Crystal structure of an IdeR-DNA complex reveals a conformational change in activated IdeR for base-specific interactions, J. Mol. Biol. 342, 1155-1169.

7. Feese, M. D., Ingason, B. P., Goranson-Siekierke, J., Holmes, R. K., and Hol, W. G. (2001) Crystal structure of the iron-dependent 
regulator from Mycobacterium tuberculosis at 2.0-A resolution reveals the Src homology domain 3-like fold and metal binding function of the third domain, J. Biol. Chem. 276, 5959-66.

8. Rangachari, V., Marin, V., Bienkiewicz, E. A., Semavina, M. Guerrero, L., Love, J. F., Murphy, J. R., and Logan, T. M. (2005) Sequence of ligand binding and structure change in the diphtheria toxin repressor upon activation by divalent transition metals, Biochemistry 44, 5672-5682.

9. Ding, X., Zeng, H., Schiering, N., Ringe, D., and Murphy, J. R. (1996) Identification of the primary metal ion-activation sites of the diphtheria tox repressor by X-ray crystallography and sitedirected mutagenesis, Nat. Struct. Biol. 3, 382-387.

10. Pohl, E., Holmes, R. K., and Hol, W. G. J. (1999) Crystal structure of the iron-dependent regulator (IdeR) from Mycobacterium tuberculosis shows both metal binding sites fully occupied, J. Mol. Biol. 285, 1145-1156.

11. Pohl, E., Holmes, R. K., and Hol, W. G. J. (1999) Crystal structure of a cobalt-activated diphtheria toxin repressor-DNA complex reveals a metal-binding SH3-like domain, J. Mol. Biol. 292, 653667.

12. Twigg, P. D., Parthasarathy, G., Guerrero, L., Logan, T. M., and Caspar, D. L. (2001) Disordered to ordered folding in the regulation of diphtheria toxin repressor activity, Proc. Natl. Acad. Sci. U.S.A. 98, 11259-11264.

13. Spiering, M. M., Ringe, D., Murphy, J. R., and Marletta, M. A. (2003) Metal stoichiometry and functional studies of the diphtheria toxin repressor, Proc. Natl. Acad. Sci. U.S.A. 100, 3808-3813.

14. Edelhoch, H. (1967) Spectroscopic determination of tryptophan and tyrosine in proteins, Biochemistry 6, 1948-1954.

15. Ellman, G. L. (1959) Tissue sulfhydryl groups, Arch. Biochem. Biophys. 82, 70-77.

16. Chou, C. J., Wisedchaisri, G., Monfeli, R. R., Oram, D. M. Holmes, R. K., Hol, W. G., and Beeson, C. (2004) Functional studies of the Mycobacterium tuberculosis iron-dependent regulator, J. Biol. Chem. 279, 53554-53561.

17. Tao, X., Zeng, H. Y., and Murphy, J. R. (1995) Transition metal ion activation of DNA binding by the diptheria tox repressor requires the formation of stable homodimers, Proc. Natl. Acad. Sci. U.S.A. 92, 6803-6807.

18. Nakamoto, K. (1960) Ultraviolet spectra and structures of 2,2'bipyridine and $2,2^{\prime}, 2^{\prime \prime}$-terpyridine in aqueous solution, J. Phys. Chem. 64, 1420-1425.

19. Holyer, R. H., Hubbard, C. D., Kettle, S. F. A., and Wilkins, R. G. (1966) The kinetics of replacement reactions of complexes of the transition metals with $2,2^{\prime}, 2^{\prime \prime}$-terpyridine, Inorg. Chem. 5 , $622-625$

20. Johnson, M. L., Correia, J. J., Yphantis, D. A., and Halvorson, H. R. (1981) Analysis of data from the analytical ultracentrifuge by nonlinear least-squares techniques, Biophys. J. 36, 575-588.

21. Tao, X., Zeng, H. Y., and Murphy, J. R. (1995) Transition metal ion activation of DNA binding by the diphtheria tox repressor requires the formation of stable homodimers, PNAS 92, 68036807.

22. Winzor, D. J., and Sawyer, W. H. (1995) Quantitative Characterization of Ligand Binding, J. Wiley, New York.

23. Dahlquist, F. W. (1978) The Meaning of Scatchard and Hill Plots, Methods in Enzymology, pp 270-299, Academic Press, New York.

24. Qiu, X., Verlinde, C. L. M. J., Zhang, S., Schmitt, M. P., Holmes, R. K., and Hol, W. G. J. (1995) Three-dimensional structure of the diphtheria toxin repressor in complex with divalent cation corepressors, Structure 3, 87-100.

25. Qiu, X., Pohl, E., Holmes, R. K., and Hol, W. G. J. (1996) Highresolution structure of the diptheria toxin repressor complexed with cobalt and manganese reveals an SH3-like third domain and suggests a possible role of phosphate as co-corepressor, Biochemistry 35, 12292-12302.

26. Pohl, E., Qiu, W., Must, L. M., Holmes, R. K., and Hol, W. G. J. (1997) Comparison of high-resolution structures of the diphtheria toxin repressor in complex with cobalt and zinc at the cationanion binding site, Protein Sci. 6, 1114-1118.

27. Goranson-Siekierke, J., Pohl, E., Hol, W. G. J., and Holmes, R. K. (1999) Anion-coordinating residues at binding site 1 are essential for the biological activity of the diphtheria toxin repressor, Infect. Immun. 67, 1806-1811.

28. Love, J. F., vanderSpek, J. C., Marin, V., Guerrero, L., Logan, T. M., and Murphy, J. R. (2004) Genetic and biophysical studies of diphtheria toxin repressor (DtxR) and the hyperactive mutant
DtxR(E175K) support a multistep model of activation, Proc. Natl. Acad. Sci. U.S.A. 101, 2506-2511.

29. Love, J. F., VanderSpek, J. C., and Murphy, J. R. (2003) The src homology 3-like domain of the diphtheria toxin repressor (DtxR) modulates repressor activation through interaction with the ancillary metal ion-binding site, J. Bacteriol. 185, 2251-2258.

30. Rangachari, V., Marin, V., Bienkiewicz, E. A., Semavina, M., Guerrero, L., Love, J. F., Murphy, J. R., and Logan, T. M. (2005) Sequence of ligand binding and structure change in the diphtheria toxin repressor upon activation by divalent transition metals, Biochemistry 44, 5672-5682.

31. D’Aquino, J. A., Tetenbaum-Novatt, J., White, A., Berkovitch, F., and Ringe, D. (2005) Mechanism of metal ion activation of the diphtheria toxin repressor DtxR, PNAS 102, 18408-18413.

32. Monod, J., Wyman, J., and Changeux, J.-P. (1965) On the nature of allosteric transitions: A plausible mode, J. Mol. Biol. 12, 88118.

33. Cantor, C. R., and Schimmel, P. R. (1980) The Behavior of Biological Macromolecules, in Biophysical Chemistry (McCombs, L. W., Ed.) pp 859-866, W. H. Freeman and Co., San Francisco, CA.

34. Lieser, S. A., Davis, T. C., Helmann, J. D., and Cohen, S. M. (2003) DNA-binding and oligomerization studies of the manganese(II) metalloregulatory protein MntR from Bacillus subtilis, Biochemistry 42, 12634-12642.

35. Bell, S. D., Cairns, S. S., Robson, R. L., and Jackson, S. P. (1999) Transcriptional regulation of an archaeal operon in vivo and in vitro, Mol. Cell 4, 971-982.

36. Hantke, K. (2001) Iron and metal regulation in bacteria, Curr. Opin. Microbiol. 4, 172-177.

37. Mills, S. A., and Marletta, M. A. (2005) Metal binding characteristics and role of iron oxidation in the ferric uptake regulator from Escherichia coli, Biochemistry 44, 13553-13559.

38. Pecqueur, L., D' Autreaux, B., Dupuy, J., Nicolet, Y., Jacquamet, L., Brutscher, B., Michaud-Soret, I., and Bersch, B. (2006) Structural changes of E. coli fur during metal dependent dimerization and activation explored by NMR and X-ray crystallography, J. Biol. Chem. M601278200.

39. Dosanjh, N. S., and Michel, S. L. J. (2006) Microbial nickel metalloregulation: NikRs for nickel ions, Curr. Opin. Chem. Biol. $10,123-130$.

40. Wang, Y., Hemmingsen, L., and Giedroc, D. P. (2005) Structural and functional characterization of Mycobacterium tuberculosis $\mathrm{CmtR}$, a Pb/ $\mathrm{Cd}^{\mathrm{II}}$-sensing $\mathrm{SmtB} /$ ArsR metalloregulatory repressor, Biochemistry 44, 8976-8988.

41. Zeng, Q., Stalhandske, C., Anderson, M. C., Scott, R. A., and Summers, A. O. (1998) The core metal-recognition domain of MerR, Biochemistry 37, 15885-15895.

42. Fraczkiewicz, R., Braun, W. (1998) Exact and efficient analytical calculation of the accessible surface areas and their gradients for macromolecules, J. Comput. Chem. 19, 319-333.

43. Good, N. E., Winget, G. D., Winter, W., Connolly, T. N., Izawa, S, and Singh, R. M. M. (1966) Hydrogen ion buffers for biological research, Biochemistry 5, 467-477.

44. Choe, W.-S., Clemmitt, R. H., Chase, H. A., and Middelberg, A. P. J. (2003) Coupling of chemical extraction and expanded-bed adsorption for simplified inclusion-body processing: Optimization using surface plasmon resonance, Biotechnol. Bioeng. 81, 221232 .

45. Zhou, H.-X. (2004) Protein folding and binding in confined spaces and in crowded solutions, J. Mol. Recognit. 17, 368-375.

46. Finney, L. A., and O'Halloran, T. V. (2003) Transition metal speciation in the cell: Insights from the chemistry of metal ion receptors, Science 300, 931-936.

47. Guedon, E., and Helmann, J. D. (2003) Origins of metal ion selectivity in the DtxR/MntR family of metalloregulators, Mol. Microbiol. 48, 495-506.

48. Koradi, R., Billeter, M., and Wüthrich, K. (1996) MOLMOL: A program for display and analysis of macromolecular structures, J. Mol. Graphics 14, 51-55.

BI060797S 